\title{
L'arabe marocain au contact du français sur les réseaux sociaux numériques diasporiques: quand deux langues se rivalisent
}

\author{
Moroccan Arabic in contact with French on diasporic \\ digital social networks: when two languages compete
}

\author{
Mourad El Baroudi \\ Université Sidi Mohammed Ben Abdallah de Fès (Maroc) \\ baroudimourad@gmail.com
}

\begin{abstract}
The aim of this article is to focus on the language practices of the Moroccan diaspora in contact situations on digital social networks. Indeed, the members of this online community, in their written language interactions on these digital universes, show a remarkable creativity when they mix Moroccan Arabic and French; They use specific typographical marks and change the matrix language from one statement to another. Moreover, these bilingual language practices are the product of a crossbreeding between these practices in their oral form and the technology that has given them a written form. Therefore, the data of our study will be not only the language elements, i.e. the statements, but also the composite elements that cross cultural, identity, social and technological aspects.
\end{abstract}

\section{Key-words}

Digital social networks, Moroccan diaspora online, bilingual language practices, matrix language.

\section{Resumen}

El objetivo de este artículo es examinar las prácticas lingüísticas de la diáspora marroquí en situaciones de contacto en las redes sociales digitales. De hecho, los miembros de esta comunidad en línea, en sus interacciones lingüísticas escritas en estos universos digitales, muestran una notable creatividad cuando mezclan el árabe marroquí y el francés; Utilizan marcas tipográficas específicas y cambian el lenguaje de la matriz de una declaración a otra. Además, estas prácticas lingüísticas bilingües son el producto de una fertilización cruzada entre estas prácticas en su forma oral y la tecnología que les ha dado una forma escrita. Por tanto, los datos de nuestro estudio no serán sólo los elementos lingüísticos, es decir, los enunciados, sino también los elementos compuestos que atraviesan aspectos culturales, identitarios, sociales y tecnológicos.

\section{Palabras clave}

Redes sociales digitales, diáspora marroquí en línea, prácticas lingüísticas bilingües, lengua matriz. 


\section{Introduction}

La diaspora marocaine en ligne, dans ses interactions langagières écrites sur les réseaux sociaux numériques (désormais RSN), fait preuve d'une ingéniosité remarquable quand elle mélange l'arabe marocain et le français. Pour réussir ce mélange dans son état écrit, les membres de cette diaspora recourent à des marques typographiques spécifiques (chiffres, rébus, néographies...). En outre, ils ont tendance à changer la langue matrice d'un énoncé à un autre; dans certains cas, l'arabe marocain joue le rôle de la langue matrice ${ }^{1}$, dans d'autres cas, c'est le français qui assure ce rôle. Il s'agit donc d'une sorte de concurrence entre les deux langues générant des phénomènes linguistiques inédits.

L'objectif de cet article est d'interroger ces pratiques langagières de la diaspora marocaine en situation de contact sur les réseaux sociaux numériques. En fait, ces pratiques langagières bilingues n'existaient pas dans leur forme écrite avant l'avènement de la technologie du web 2.0. Elles sont nées et se sont développées dans les univers numériques. Elles sont donc le produit d'un métissage entre ces pratiques dans leur forme orale et la technologie qui leur a offert une forme écrite et un environnement de production spécifique. De ce fait, les données de notre étude seront non seulement des éléments langagiers à savoir les énoncés, mais aussi d'autres éléments qui relèvent de leur écosystème de production et qui croisent des aspects culturel, identitaire, social et technologique.

Dans un premier temps, nous présenterons notre corpus ainsi que le cadre théorique et méthodologique adopté. Dans un deuxième temps, nous nous interrogerons sur ces pratiques langagières de la diaspora marocaine en ligne sur les RSN en posant principalement les questions suivantes: ces pratiques langagières représentent-elles un seul système du français ou deux systèmes et deux codes linguistiques différents? Autrement dit, les membres de cette communauté en ligne font-ils de simples emprunts à la langue d'origine, l'arabe, ou manipulent-ils deux langues dans leurs interactions numériques écrites? Quelles sont les spécificités typographiques et linguistiques de cette multitude de codes? Dans quelle position ces pratiques langagières bilingues écrites sur les RSN situent-elles la diaspora marocaine par rapport à leurs deux communautés, d'origine et d'accueil?

Toutes ces interrogations traduisent la complexité de l'analyse de ces pratiques langagières qui imbriquent plusieurs aspects: (1) typographique: quelle graphie utilisée? (2) linguistique: quel codeadopté? (3) extralinguistique: quelle influence des facteurs sociaux, culturels, identitaires et (4) technologique: quel impact des constituants de l'environnement numérique?

1 La langue matrice est celle qui fournit le cadre morphosyntaxique de l'énoncé, contrairement à la langue enchâssée, qui y participe par des morphèmes lexicaux (Myers-Scotton, 2002). 


\section{Bref rappel sociohistorique de cette diaspora}

Les Marocains résidant à l'étranger sont estimés à 4,5 millions de personnes dont un tiers vit en France ${ }^{2}$. Cette grande présence des Marocains en France n'est pas un phénomène récent. Les flux migratoires vers la France datent d'un peu d'un siècle et ont pris plusieurs formes: la migration de la main-d'œuvre, la migration familiale, la migration illégale, la migration étudiante et la migration des compétences.

Plusieurs appellations sont utilisées pour désigner cette communauté: "Ressortissants Marocains à l'Étranger” (RME), "Marocains Résidants à L'Étranger” (MRE), "Marocains du monde" ou encore les "binationaux". Le terme "diaspora" est quant à lui surtout utilisé "lorsqu'il s'agit de rendre compte de toutes les formes de liens existant entre des Marocains résidant à l'étranger et leur pays d'origine" (Alaoui 2013:7).

Ce lien avec le pays d'origine se manifeste surtout par des retours annuels pour passer les vacances d'été, par les transferts d'argents et par l'intérêt porté à la politique étrangère du Maroc, à son progrès économique et à l'équipe nationale du football.

Le maintien des liens culturels et cultuels, constitue un autre élément de cet attachement, notamment grâce à l'enseignement de la langue arabe et de la culture marocaine (ELCO) dans les écoles françaises, à l'envoi des imams en France, aux programmes télévisés émis par la chaîne Al-maghribia, aux sites internet diasporiques comme le site Yabiladi et également grâce aux RSN dont fait partie le groupe Facebook diasporique "Marocain de France" objet de notre étude.

\section{Arrière-plan théorique}

Nos lectures dans la littérature de l'analyse du discours numérique nous ont permis de distinguer deux étapes essentielles dans l'évolution des approches dans ce domaine:

- Les anciennes perspectives appelées “internalistes" (Develotte \& Paveau 2017: 200) se limitaient à l'analyse des données extraites sur internet sans prendre en compte l'environnement de leurs productions.

- Les nouvelles perspectives dites “externalistes” (ibid.: 201) s'orientent de plus en plus vers la prise en considération de tout l'environnement de production de ces données, en considérant le discours numérique comme étant un discours composite, multimodal et évolutif.

Dans la présente étude, nous ferons référence à ces nouvelles perspectives car il nous semble qu'elles soient plus appropriées pour apporter des réponses à notre terrain caractérisé

2 Selon les chiffres officiels, en 2012, 3,4 millions de MRE (juifs d'origine marocaine non compris) résident à l'étranger. Un tiers (soit 1146000 personnes) vit en France. (Statistiques de la DACS, Direction des Affaires Consulaires et Sociales du Ministère des Affaires Sociales, tirées de 1'article de Alaoui (2013:12)) 
par la complexité des pratiques langagières liées à la fois à l'utilisation de la technologie numérique et à la diversité des codes employés.

Parmi ces perspectives, l'approche écologique ou environnementale de Marie-Anne Paveau qui repose sur la notion de "l'environnement" paraît être la plus pertinente pour répondre à nos questions. Selon elle,

L'environnement est l'ensemble des données humaines et non humaines au sein desquelles les discours sont élaborés. Ces données concernent tous les domaines de l'existence: elles sont sociales, culturelles, historiques, matérielles, animales, naturelles, etc. (Paveau, 2013)

Ainsi,

Les observables de l'analyse ne sont plus seulement des éléments purement langagiers, mais des matières composites, métissées de social, de culturel, d'historique, etc. [...], mais aussi de technologique. (Paveau 2014: 3)

En nous référant à cette approche, nous essaierons d'appréhender la manière dont les paramètres extralinguistiques (facteurs culturels, identitaires et sociaux) et les affordances $^{3}$ de l'environnement numérique conditionnent les pratiques langagières bilingues de la diaspora marocaine sur les RSN et d'identifier les phénomènes linguistiques qui en découlent.

Plus précisément, nous nous intéresserons dans cet article principalement aux pratiques langagières bilingues en tenant compte de leur environnement numérique de production. Le traitement des données multimodales sera limité à rendre compte des conditions d'apparition des interactions dans ce groupe et de certains faits linguistiques qui résultent du contact entre le français et l'arabe marocain.

Cela étant, il va sans dire que nous n'allons pouvoir traiter la totalité des données multimodales et des éléments techno-discursifs présents dans cet espace numérique. Un travail sur cette matière pourrait en effet faire, à lui seul, l'objet d'un autre article et permettrait d'exploiter la totalité de l'environnement discursif de cet espace numérique diasporique.

\section{Corpus et sa construction}

Notre étude sera fondée sur un "corpus idionumérique" (Émerit, 2016: 22), tiré d'un groupe Facebook intitulé "Marocains de France" qui rassemble plus de 94000 membres étant en principe, comme l'intitulé du groupe l'indique, des Marocains de France. Ils sont

3 Utilisée pour la première fois par Gibson (1979) dans le champ de la psychologie sociale, la notion d'affordance désigne l'ensemble des potentialités d'actions offertes par un environnement. Dans notre champ d'étude, on désigne par "affordances numériques" toutes les actions permises par les outils technologiques (ordinateur, smartphone, clavier, écran, etc.) et les environnements numériques (comme les différentes fonctionnalités de Facebook). 
très actifs sur ce groupe. A titre d'exemple, le 31 mai 2021 à 22 heures, on compte 27 nouvelles publications et 962 publications en mois d'avril. La plupart des publications reçoivent des centaines de réactions et de commentaires.

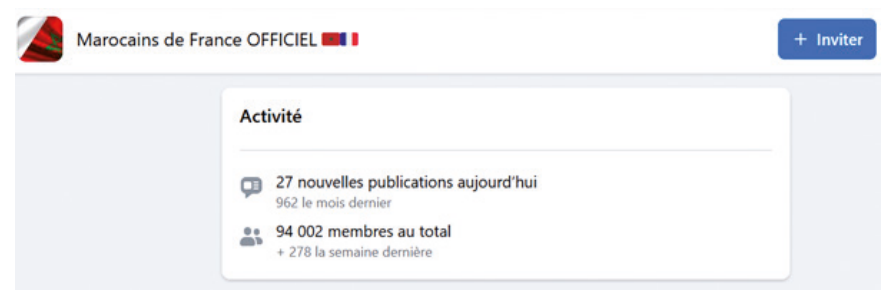

Fig. 1: Capture d'écran prise le 31 mai 2021, montrant le nombre des membres sur le groupe Facebook "Marocains de France OFFICIEL"

Cependant, quand on se trouve en face d'un corpus numérique, la question de sa construction s'impose vu certaines spécificités de ce discours. Il est multimodal instable et évolutif. Pour résoudre ce problème, nous avons choisi de faire appel à la notion du "corpus idionumérique". Grâce à une représentation arborescente, ce modèle vise à prendre en considération le caractère continu et évolutif des données qui apparaissent dans les corpus 2.0. Il propose d'effectuer plusieurs niveaux d'extractions selon un ordre arborescent.

Nous utiliserons ce modèle pour présenter notre corpus tout en adaptant les niveaux d'extractions aux besoins de notre étude. Ainsi, la représentation arborescente de notre corpus contient quatre niveaux d'extractions représentés dans ce schéma:

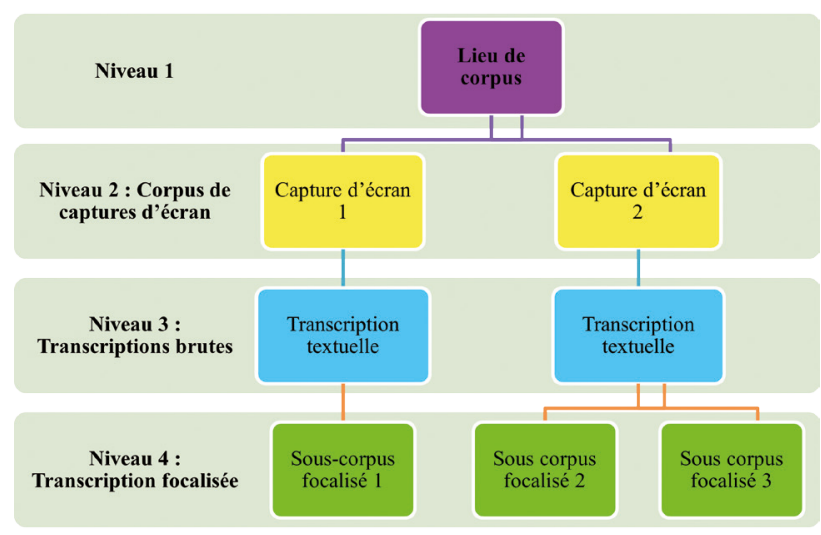

Fig. 2: Représentation arborescente à quatre niveaux d'extraction.

4 L'intitulé de ce groupe précise "Officiel" pour se distinguer d'autres groupes qui portent un intitulé proche de celui-ci, mais qui n'ont pas rencontré le même succès: le groupe "les marocains de France" qui compte 4788 membres, le groupe "Marocains de France, publicités et annonces" qui contient 3558 membres et une page intitulée "Marocain, marocaine de France" qui a 2952 abonnés. 
Anales de Filología Francesa, n. ${ }^{\circ}$ 29, 2021

L'ARABE MAROCAIN AU CONTACT DU FRANÇAIS SUR LES RÉSEAUX SOCIAUX NUMÉRIQUES...

La représentation de notre corpus sera donc comme suit:

Niveau 1: Lieu de corpus.

Il s'agit du groupe Facebook intitulé "Marocains de France OFFICIEL", consultable uniquement en ligne en suivant ce lien: < https://www.facebook.com/groups/669297839760760>. Il constitue la source de toutes les données.

Niveau 2: Corpus des captures d'écrans.

Les captures d'écrans sont les instantanés du lieu de corpus pris à un moment donné et focalisés sur un ou des phénomènes précis. Le corpus à ce niveau-là est difficile à exploiter à cause du grand nombre de données qu'il contient. Mais, il est très important dans la mesure où il offre à la fois des informations indispensables pour l'analyse des énoncés et une base de travail stable à partir de laquelle on peut extraire des données textuelles exploitables linguistiquement.

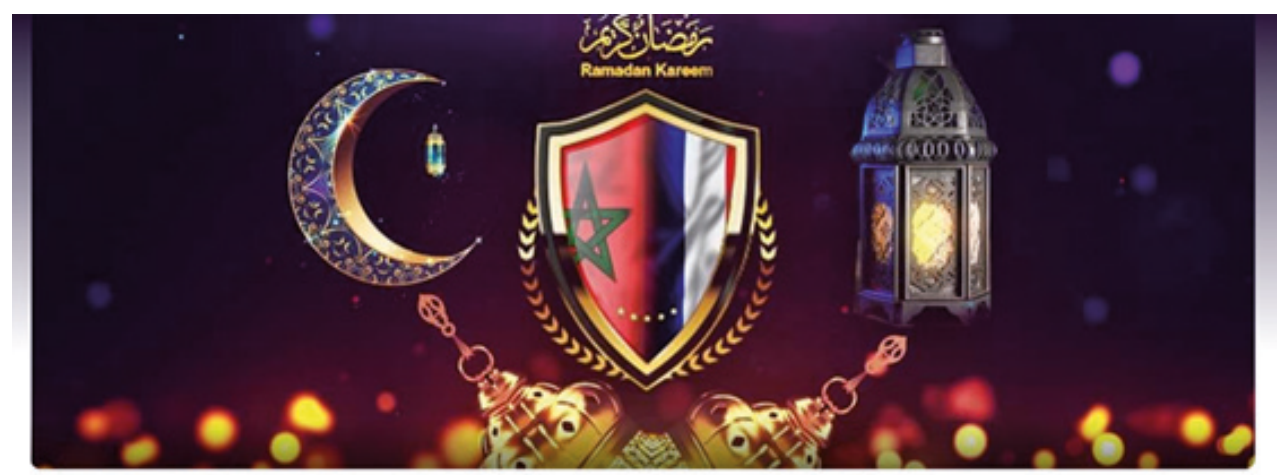

\section{Marocains de France OFFICIEL}

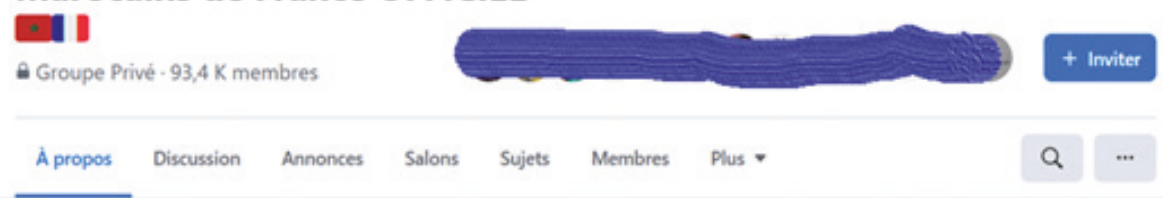

Fig. 3: Instantané du lieu de corpus pris le 31 mai 2021 relevant du niveau 2 de la représentation et focalisé sur la couverture du Groupe.

Par exemple, la capture d'écran ci-dessus prise le 31 mai 2021, représentant un instantané du lieu de corpus focalisé sur la couverture du Groupe, nous donne plusieurs informa- 
tions sur le contenu de ce groupe entre autres: l'intitulé "Marocains de France OFFICIEL", les drapeaux du Maroc et de la France représentent un ancrage identitaire, la photo de couverture représentant quelques aspects culturels du pays d'origine. En effet, l'administrateur du groupe a changé la couverture du groupe à l'occasion du mois du ramadan. Sur la photo, on distingue les motifs suivants qui représentent les festivités de ce mois sacré: le fanous (la lanterne), le croissant de la lune et deux encensoirs. De plus, les couleurs faisant référence à la nuit contribuent à créer l'atmosphère des soirées animées de ce mois. On note aussi la présence en tête de la photo de couverture de l'expression Ramadan Kareem (bon ramadan) en graphies arabe et latine.

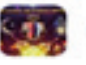

Marocains de France OFFICIEL

\section{+ Inviter

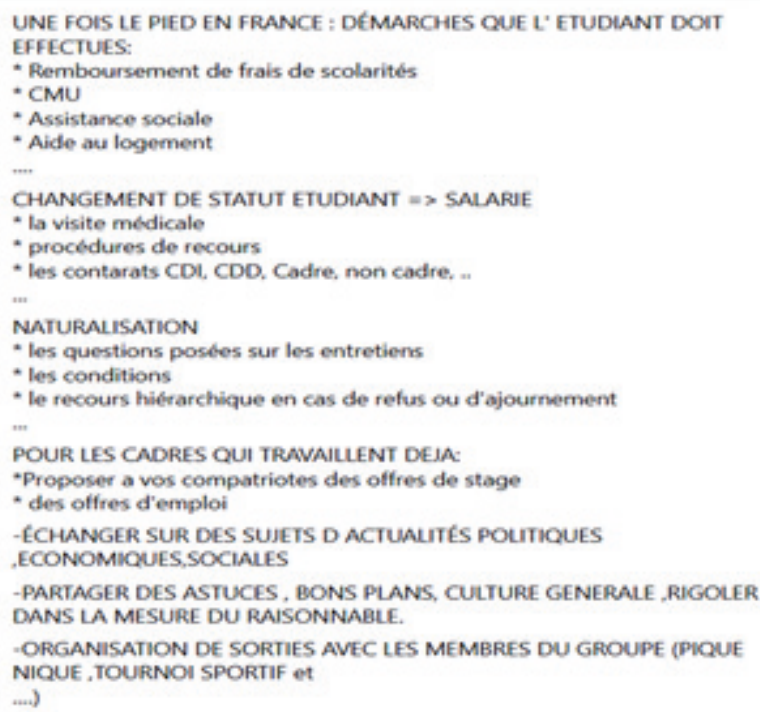

Fig. 4: Instantané du lieu de corpus relevant du niveau 2 de la représentation et focalisé sur la rubrique "à propos".

De même, cette capture d'écran étale les objectifs de ce groupe. Nous trouvons par exemple:

- Solliciter de l'aide face aux problèmes rencontrés dans le pays d'accueil

- Chercher des informations pratiques liées au Maroc ou à la France (naturalisation, réussir un voyage au Maroc).

- Débattre de sujets politiques, sociaux et religieux, etc.

Les captures d'écran à ce niveau-là peuvent aussi offrir au chercheur une base de travail stable sans laquelle un travail de recherche en linguistique semble impossible. 


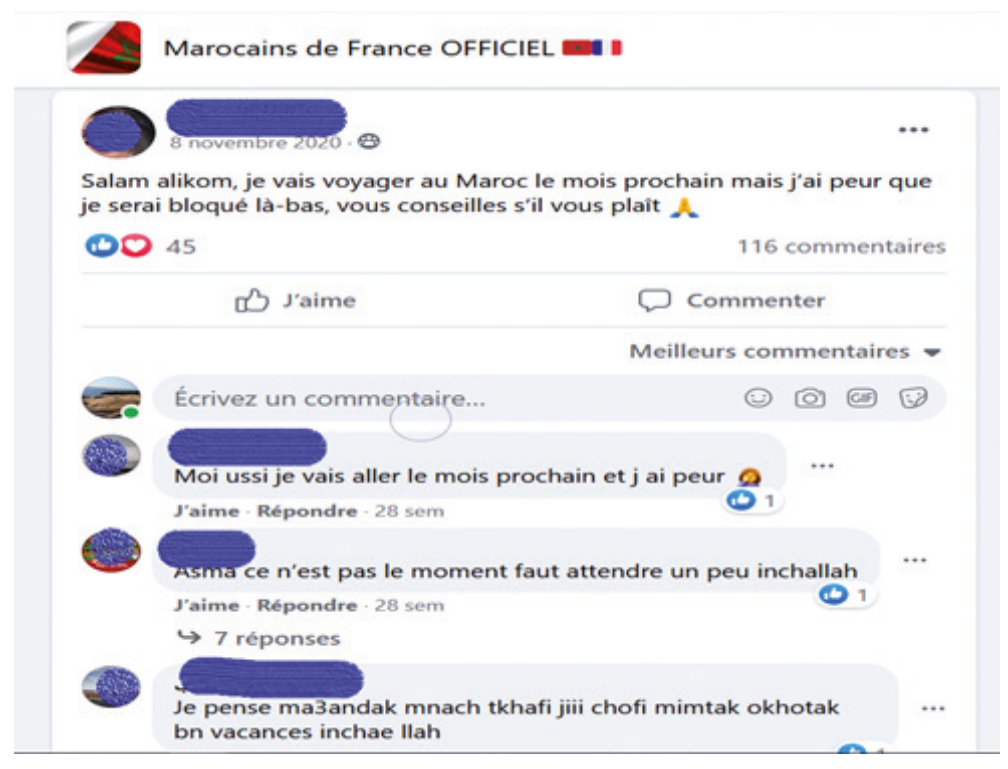

Fig. 5: Capture d'écran représentant des cas de contact entre l'arabe marocain et le français.

À partir de ces représentations figées, on peut extraire différents corpus focalisés sur un objet donné en fonction de la problématique traitée. Dans le cadre de notre corpus, nous avons notamment réalisé des captures d'écran focalisées sur des cas de contact entre l'arabe marocain et le français.

\section{Niveau 3: Transcriptions brutes.}

Ce sont des transcriptions brutes des données du niveau 2. À ce stade, les données sont de nature textuelle et peuvent donc être analysées au niveau linguistique. Il faut noter néanmoins la perte de quelques éléments techno-langagiers comme les images et les couleurs. Seules les données textuelles sont préservées. Si l'on reprend l'exemple de la figure 5, on extrait les données textuelles suivantes:

\section{Locuteur 1}

8 novembre 2020

Salam alikom, je vais voyager au Maroc le mois prochain mais j'ai peur que je serai bloqué là-bas, vous conseilles s'il vous plaît

116 commentaires

Meilleurs commentaires

Locuteur 2

Moi ussi je vais aller le mois prochain et $j$ ai peur

J'aime Répondre 28 sem 
Anales de Filología Francesa, n. ${ }^{\circ}$ 29, 2021

MOURAD EL BAROUDI

Locuteur 3

Asma ce n'est pas le moment faut attendre un peu inchallah

J'aime Répondre 28 sem

7 réponses

Locuteur 4

Je pense ma3andak mnach tkhafi jiii chofi mimtak okhotak bn vacances inchae llah

Niveau 4: Transcriptions focalisées.

Il s'agit des sous-catégories contenant seulement quelques éléments de corpus en fonction de la focalisation de recherche. Elles sont de nature textuelle, composées des énoncés susceptibles d'être analysés au niveau linguistique après une transcription et une traduction.

Par exemple, à partir des données textuelles précédentes, nous relevons les énoncés suivants représentant des cas de contact entre l'arabe marocain et le français et qui sont prêts à être soumis à une analyse linguistique:

(1) Salam alikom [notre traduction: que la paix soit sur vous (salut)], je vais voyager au Maroc le mois prochain mais j'ai peur que je serai bloqué là-bas, vous conseilles s'il vous plaît.

(2) Asma ce n'est pas le moment faut attendre un peu inchallah [notre traduction: si Dieu le veut].

(3) Je pense ma3andak mnach tkhafi jiii chofi mimtak okhotak [notre traduction: tu n'as pas de quoi avoir peur. Viens voir ta mère et tes frères !] bn vacances inchae llah [si Dieu le veut].

Concrètement, cette représentation du corpus selon une cartographie hiérarchisée en 4 niveaux permet d'une part de résoudre le problème de l'instabilité du corpus numérique et d'autre part d'analyser linguistiquement les données tout en assurant leur traçabilité et en prenant en compte les conditions de leur production.

Par ailleurs, étant donné que nous avons affaire à un "Lieu de corpus" gigantesque, instable et évolutif, nous ne pouvons pas présenter des statistiques fiables sur la répartition des différentes langues ni de donner une portée chiffrée pour chaque phénomène linguistique observé. Pour parer à cette difficulté, nous avons parcouru la rubrique "Discussion" de ce groupe Facebook et nous avons effectué 200 captures d'écran relevant du Niveau 2 de la représentation. Parmi ces captures, 12 sont présentées dans la section annexe puis nous avons procédé à une analyse des données textuelles relevant des niveaux 3 et 4 pour établir les occurrences et les répartitions des différents phénomènes linguistiques observés. 


\section{Résultats et discussion}

À travers l'étude des énoncés puisés dans notre lieu de corpus qui relève du niveau 4 de la représentation ${ }^{5}$, nous essaierons d'abord d'appréhender la question du choix de langues (français, arabe marocain) et de l'alternance codique. Ensuite, nous aborderons les caractéristiques typographiques de leur écriture sur les RSN. Enfin, nous examinerons la spécificité linguistique de l'alternance codique telle qu'elle est pratiquée par cette communauté sur ce Groupe Facebook diasporique.

\subsection{Quelles sont les langues utilisées dans le Groupe Facebook diasporique "Marocains de France"?}

L'observation des interactions langagières dans ce Groupe nous a montré que les énoncés sont soit en français, soit en arabe marocain, soit formés d'un mélange des deux langues. En effet, le français est présent considérablement dans les interactions de ce groupe. Il est très sollicité par les usagers. Ce choix pourrait être justifié par le fait que le français est la langue de travail ou d'étude de la plupart des membres (en majorité, des migrants marocains vivants en France, des étudiants en France ou des Marocains nés en France). On peut supposer donc que l'utilisation du français sur cet environnement numérique est spontanée surtout quand il s'agit d'évoquer des sujets en relation avec les domaines du travail ou des études.

L'arabe marocain est également présent dans ce groupe Facebook diasporique mais à un degré moindre que le français. Les usagers utilisent principalement le clavier latin pour transcrire leurs interactions dans cette langue. Ce choix est justifié par le fait que la plupart des locuteurs de ce groupe ont l'habitude d'utiliser le clavier latin dans le cadre de leur travail ou de leurs études. Le clavier arabe est également utilisé, mais moins souvent. Le recours à l'arabe marocain facilite à notre avis la communication et permet aux locuteurs de se détacher des contraintes des langues, car n'ayant pas d'orthographe codifiée, il permet de communiquer librement sans s'inquiéter de commettre des erreurs de grammaire ou d'orthographe. Ainsi, dans le corpus, on trouve plusieurs orthographes en graphie latine du même mot arabe:

Exemples:

Salam aleikom (illustration 7)/Salam alaykum (Illustration 4) "que la paix soit sur vous"

lmghrib (illustration 4)/l Maghreb (Illustration 8) "le Maroc"

inchae llah/in chaalah (Illustration 6) "si Dieu le veut"

chakhsiya/chakhssia (Illustration 8) "la personnalité"

5 Comme nous l'avons mentionné auparavant, il s'agit des transcriptions focalisées sur des cas de contact entre l'arabe marocain et le français. 
Il est à souligner que l'arabe standard est peu présent. On remarque, par exemple, que sa présence est plus importante lors d'échanges en relation avec le domaine de la religion. En effet, la majorité des membres de cette communauté s'identifient à la culture musulmane. Cela se manifeste dans leurs interactions par l'emploi des expressions comme inchae llah "si Dieu le veut", salamoualikoum "que la paix soit sur vous", hamdoulillah "Dieu soit loué".

Nous signalons également une présence faible du berbère, de l'espagnol et de l'anglais qui pourrait être due au fait que les répertoires linguistiques des membres de cette diaspora sont différents ainsi qu'au fait qu'ils ne sont pas tous compétents dans ces langues. En effet, la langue maternelle des Marocains change selon qu'ils sont arabophones ou berbérophones. De plus, la deuxième langue étrangère, après le français ${ }^{6}$, apprise à l'école marocaine peut être l'anglais ou l'espagnole selon qu'ils sont originaires du nord, du centre ou du sud du Maroc.

Pour ce qui est de l'alternance codique, elle a une présence massive dans ce Groupe diasporique. Cette pratique qui intègre le français et l'arabe dans un même énoncé n'est pas nouvelle. Elle s'inscrit dans l'évolution de ces pratiques dans leur forme orale car la diaspora marocaine pratiquait l'alternance codique, avant l'avènement du web 2.0, dans leurs communications quotidiennes, entre eux, dans des situations de face-à-face. Ainsi, les fonctions de l'alternance codique dans ce groupe Facebook diasporique ne s'écartent pas à notre avis "des fonctions classiques observées par les sociolinguistes: interactionnelles, sociales et psychologiques" (Atifi, 2007: 123). Généralement, son apparition, comme le souligne M. Darot, “dépend de l'image que le locuteur se fait de l'interlocuteur" (1995: 121).

En somme, l'observation des interactions langagières dans le Groupe diasporique "Marocains de France" nous a permis de dégager la façon dont les Marocains de France gèrent l'utilisation d'une partie de leurs répertoires linguistiques. Ces derniers, qui résident en France, mais ne partagent pas les mêmes conditions d'acquisition de l'arabe marocain et du français, alternent des usages monolingues en français ou en arabe marocain, tenus séparément, avec des usages mixtes d'unités linguistiques françaises et arabes. Ainsi, ils abolissent toutes les frontières séparant les deux langues.

\subsection{Quelques spécificités typographiques de cette multitude de codes}

Pour réussir le mélange de deux langues l'arabe marocain et le français de graphies différentes, les usagers sur les RSN recourent à une typographie particulière. On note notamment le recours à des chiffres pour transcrire des lettres arabes qui n'existent pas dans l'alphabet latin. Le/ḥ/= ح, le/q/= ق $, 1 \mathrm{e} / \mathrm{P} /=\varsigma, 1 \mathrm{e} / \varepsilon /=\varepsilon$ sont transcrits respectivement par les chiffres 7, 9, 2, 3 par ressemblance aux lettres arabes ${ }^{7}$.

6 Le français est la première langue étrangère du Maroc. Son apprentissage est obligatoire dans les écoles marocaines dès le CE2.

7 Nous signalons que ce système graphique trouve ses origines dans les SMS avec l'avènement des téléphones 
Anales de Filología Francesa, n. ${ }^{\circ}$ 29, 2021

L'ARABE MAROCAIN AU CONTACT DU FRANÇAIS SUR LES RÉSEAUX SOCIAUX NUMÉRIQUES...

À titre d'exemple, de l'illustration 12 ( $c f$. Annexe), on peut relever les mots suivants, qui sont transcrits en alphabet latin tout en utilisant les chiffres:

t3ich "vivre", 3aleikom "sur vous", 3alayna "sur nous", z3ma "soit disant", tab3ine "ils suivent", 3aychine "ils vivent", l9raya "les études", mas7oba "accompagnée", wa3i "conscience", 3 arfine "ils savent".

\subsection{L'alternance codique dans le Groupe Facebook diasporique "Marocains de France"}

Pour ce qui est de l'alternance codique dans le Groupe Facebook diasporique "Marocain de France", on note l'existence de trois formes: inter-phrastique, intra-phrastique et mélange des langues.

Il est à souligner que ces phénomènes linguistiques sont généralement identiques à ceux qui ont été attestés dans la littérature de l'alternance codique dans sa forme orale comme entre autres: (Abbassi, 1977; Bentahila \& Davies, 1983, 1995; Lahlou, 1991; Ziamari, 2008; Belhaiba, 2014). Sans être exhaustif, nous choisissons d'exposer les plus pertinents qui caractérisent l'alternance codique sur ce groupe Facebook diasporique en donnant plusieurs exemples puisés dans notre lieu de corpus.

Chaque exemple sera accompagné d'une note entre parenthèses le renvoyant à la capture d'écran de laquelle il est extrait. L'objectif est d'assurer la traçabilité des énoncés choisis et de rendre compte de leurs conditions de production.

\subsubsection{Alternance codique inter-phrastique}

L'alternance codique qui opère au niveau inter-phrastique et qui consiste en l'usage alternatif de deux langues (L1 et L2) distinctement dans un même énoncé se trouve très représentée dans ce groupe. Voici quelques exemples:

Exemple (1): "Il faut pas mettre tout le monde dans le même pannier (L1). Kifma kayn wlad nass kayn bnat nass w kifma kaynin bnat nass kaynin wlad nass (L2)" [notre traduction: comme il y des bons hommes il y a aussi des bonnes femmes et inversement] (cf. Annexe, Illustration 1).

Exemple (2): "Kolchi kaytsna lblad t3tih $w$ ta hed mabaghi ya3tiha chi haja... (L1) [notre traduction: tout le monde attend ce que le pays va lui offrir et personne ne veut rien offrir à son pays] c ça le problème! (L2)" (cf. Annexe, Illustration 2).

Exemple (3): 'j'ai réalisé qu'on a beaucoup de choses positives au Maroc qu'on peut jamais les trouver en Europe et que ce dernier n'est pas le paradis (L1), ghir kola o chno 3 tah lah (L2) [notre traduction: cependant, chaque pays a ses propres avantages] enfin aucun coin dans la terre n'est parfait (L1)"' (cf. Annexe, Illustration 2)

portables (Caubet, 2004), puis s'est élargi et développé sur les RSN. 


\subsubsection{Alternance codique intra-phrastique}

D'après nos observations, cette forme semble se pratiquer abondamment dans les interactions des membres de ce groupe. Elle désigne l'intégration d'une unité linguistique française dans une phrase en arabe marocain et inversement. Nous avons tenté d'examiner ces deux cas à travers plusieurs exemples:

\subsubsection{Le cas d'unités linguistiques arabes et de matrice française}

L'insertion des unités de l'arabe marocain dans une structure morphosyntaxique en français se trouve très sollicitée par les usagers de ce groupe. Cela concerne toutes sortes d'éléments de la langue, fréquemment:

- des pronoms clitiques:

Exemple (4): “ana wiyak [notre traduction: toi et moi] on ne s'entend plus". (cf. Annexe, Illustration 3).

- des indicateurs de temps et de lieu:

Exemple (5): "je voulais savoir s>il y> a un risque de fermetures des frontiéres entre le Maroc et la France men hna [d'ici] l [jusqu'à] la fin du mois de septembre" (cf. Annexe, Illustration 4).

- des articles définis, des conjonctions et des prépositions:

Exemple (6): “drt [j'ai fait] réservation d'un vol $\boldsymbol{w}$ [et] apres j'ai un empêchement wach [est-ce que ] j ai $\mathbf{l}$ [le] droit bach [pour] $\boldsymbol{n}$ annulé!? Est ce que l annulation est gratuite!?" (cf. Annexe, Illustration 5).

- des verbes:

Exemple (8): “... sinon dans la vie makan3tich [je ne donne pas] le temps lhad no3 [à ces types-là]” (cf. Annexe, Illustration 7).

- des unités appartenant au registre du domaine religieux:

Exemple (7): "Moi personnellement je n>irai pas cette année c pas le moment in chaalah [Si Dieu le veut] l'année prochaine si dieu le veut je nait pas envie d'être enfermé au Maroc" (cf. Annexe, Illustration 6).

\subsubsection{Le cas d'unités linguistiques françaises et de matrice de l'arabe marocain}

En ce qui concerne des unités françaises qui viennent s'insérer dans un énoncé en arabe marocain, on note la présence d'emprunts des mots de français qui sont intégrés tels quels par l'arabe marocain. Les cas les plus fréquents sont l'insertion des substantifs, des coordonnants, des connecteurs, des adverbes et des verbes.

Exemple 9: "hada ye3ni anaka d3if chakhsiya o 3andek no9s o maradiche b les racines dyalk. [cela veut dire que tu as une faible personnalité et tu as un manque et tu n'es pas fier de tes racines]" ( $c f$. Annexe, Illustration 8). 
Anales de Filología Francesa, n. ${ }^{\circ}$ 29, 2021

L'ARABE MAROCAIN AU CONTACT DU FRANÇAIS SUR LES RÉSEAUX SOCIAUX NUMÉRIQUES...

Exemple 10: "wa [ (interj.)] justement, jiti lfranca aji bfloussak [tu es venu.e. en France alors viens avec ton argent !]" (cf. Annexe, Illustration 9).

Exemple 11: "Hna mgharba ou tanaftakhro [Nous sommes des marocains et nous sommes fières] de l'être" (cf. Annexe, Illustration 10).

Il existe aussi des mots du français qui sont empruntés et modifiés dans leur morphologie ou dans leur syntaxe selon les exigences de l'arabe marocain. C'est le cas, par exemple, des verbes français qui sont conjugués selon les schèmes de l'arabe marocain et sont intégrés morphologiquement dans la structure de l'arabe marocain, langue matrice:

Exemple 12: "Hta ana nulawli [moi aussi on m'a annulé] le vol $w$ mchit reservet $f$ [et je suis allé.e. reserver à] Beauvais paris" (cf. Annexe, Illustration 11).

\subsubsection{Mélange du français et de l'arabe marocain}

La troisième forme de l'alternance codique est le mélange des langues. Cette forme correspond à l'intrication de ces langues au niveau d'un énoncé de sorte qu'elles ne forment qu'une seule grammaire (Belhaiba, 2014:172), rendant ainsi la reconnaissance de la langue matrice et de la langue enchâssée très difficile. C'est tantôt le français qui joue le rôle de la langue matrice tantôt l'arabe marocain qui assure ce rôle, et ce parfois dans le même énoncé.

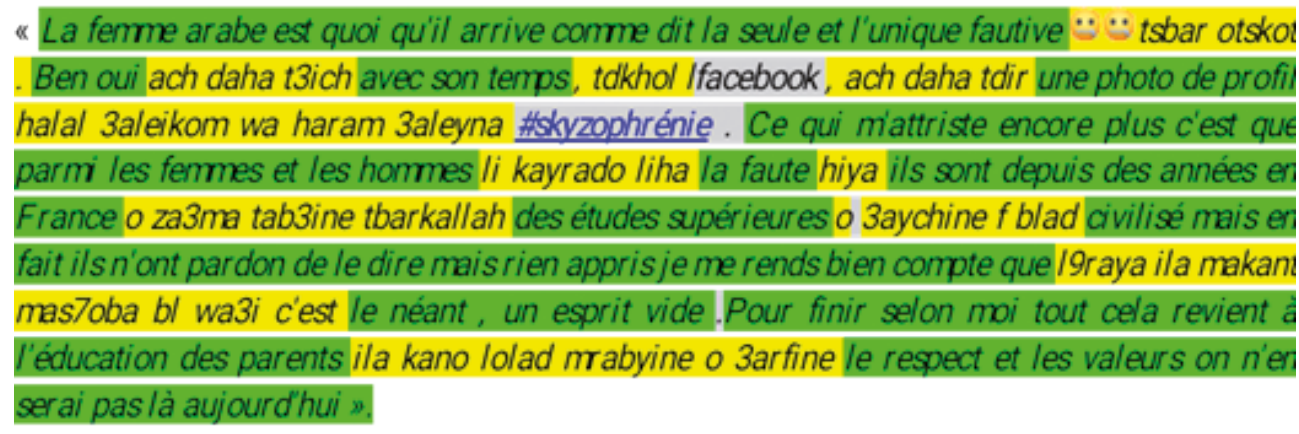

Fig. 6: Extrait d'une publication montrant l'intrication du français et de l'arabe marocain au point de ne former qu'une seule langue ${ }^{8}$ ( $c f$. Annexe, Illustration 12).

Pour exprimer ses idées dans cette publication, la locutrice puise des unités linguistiques dans son répertoire linguistique où l'arabe et le français semblent ne constituer qu'une "seule langue" (ibid.).

8 Pour faciliter les repérages des deux langues, nous avons souligné les segments de l'arabe marocain en jaune et ceux du français en vert. 


\section{Conclusion}

L'étude des pratiques langagières de la diaspora marocaine dans le groupe Facebook "Marocains de France" a montré que les membres de cette communauté possèdent des répertoires linguistiques riches et variés qui puisent de deux codes linguistiques différents l'arabe et le français. Elle nous a permis également de dégager la façon dont ces derniers utilisent leurs répertoires linguistiques dans leurs interactions langagières écrites sur ce groupe Facebook diasporique. En effet, en fonction de leur situation personnelle et des situations de communication des locuteurs qui participent à l'interaction, ils alternent des usages monolingues en français ou en arabe avec des usages mixtes produits dans les deux langues mélangées. Ces pratiques langagières évoluent également selon les contraintes de l'environnement numérique dans lequel elles sont produites.

En outre, les membres de cette communauté font preuve d'une créativité remarquable en mélangeant l'arabe marocain et le français. Pour réussir ce mélange dans son état écrit, ils recourent à des marques typographiques spécifiques. De même, les deux langues se partagent linguistiquement le rôle de langue matrice. Dans certains cas, l'arabe marocain l'emporte sur le français, dans d'autres cas le français domine structurellement l'arabe marocain.

Pour conclure, les réseaux sociaux numériques abolissant les frontières aussi bien géographiques que linguistiques facilitent le contact dans la plupart des cas plurilingues entre des communautés éloignées. C'est le cas du Groupe Facebook diasporique "Marocains de France", qui permet à la diaspora marocaine en France de s'inscrire dans les deux communautés: d'origine et d'accueil. En effet, elle se trouve insérée dans la communauté française tout en gardant le lien avec le Maroc, sa culture et ses langues.

\section{Références bibliographie}

ABbassi, Abdelaziz. 1977. A sociolinguistic analysis of multilingualism in Morocco, Austin, University of Texas. Hommes et Migrations, 1303, Diasporas marocaines.

Alaoui, Rachid. 2013. "Peut-on parler de diaspora marocaine?" in Hommes \& migrations, 1303, Diasporas marocaines: <http://journals.openedition.org/hommesmigrations/2543> [22/05/2021].

Atifi, Hassan. 2007. “Continuité et/ou rupture dans l'internet multilingue: quelles langues parler dans un forum diasporique" in Regards sur l'internet, dans ses dimensions langagières. Penser les continuités et discontinuités, Glottopol.Vol. 10, 113-126.

Belhaiba, Aicha. 2014. Le langage des jeunes issus de l'immigration maghrébine à Bordeaux. Thèse de Doctorat en Linguistique, Université Michel de Montaigne - Bordeaux III.

Bentahila, Abdelali \& Eirlys, Davies. 1983. "The syntax of Arabic-French code-switching” in Lingua 59, 331-343.

Caubet, Dominique. 2004. 'L'intrusion des téléphones portables et des 'SMS' dans l'arabe 
Anales de Filología Francesa, n. ${ }^{\circ}$ 29, 2021

L'ARABE MAROCAIN AU CONTACT DU FRANÇAIS SUR LES RÉSEAUX SOCIAUX NUMÉRIQUES...

marocain en 2002-2003" in Caubet, D., Billiez, J., Bulot, T., Leglise I. \& Miller, C. [dir.], Parlers jeunes, ici et là-bas, pratiques et représentations. Paris, L'Harmattan, 247-270.

Develotte, Christine \& Marie-Anne Paveau. 2017. "Pratiques discursives et interactionnelles en contexte numérique. Questionnements linguistiques" in Langage et société, $\mathrm{n}^{\circ} 160$ $161,199-215$.

ÉmERIT, Laetitia. 2016. "La notion de lieu de corpus: un nouvel outil pour l'étude des terrains numériques en linguistique" in Corela 14-1: <http://corela.revues.org/4594> [25/05/2021].

Gibson, James Jerome. 1979. The Ecological Approach to Visual Perception. Hillsdale, Lawrence Erlbaum.

LAHLOU, Moncef. 1991. A morpho-syntactic study of code switching between Moroccan Arabic and French. Ph. D. dissertation. The University of Texas, Austin.

LAROUSSI Foued. 1995. "L'alternance de langues arabe tunisien/français: limites de l'approche morpho-syntaxique " in MAS-GELLAS nouvelle série 7, 205-222.

Myers-Scotton, Carol. 2002. Contact linguistics: Bilingual Encounters and Grammatical Outcomes. Oxford, Oxford University Press.

Paveau, Marie-Anne. 2013. “Analyse discursive des réseaux sociaux numériques Dictionnaire d'analyse du discours numérique, Technologies discursives" in Carnet de recherche: $<$ http://technodiscours.hypotheses.org $>$ [22/05/2021].

ZIAMARI, Karima. 2008. Le code switching arabe marocain/français au Maroc: l'arabe marocain au contact du français. Paris, L'Harmattan. 
Anales de Filología Francesa, n. ${ }^{\circ}$ 29, 2021

MOURAD EL BAROUDI

\title{
Annexe
}

Corpus des captures d'écran relevant du niveau 2 de représentation et focalisées sur les cas de contact entre l'arabe marocain et le français. Elles permettent, comme nous l'avons mentionné auparavant, d'assurer la traçabilité des exemples étudiés et de reprendre compte des conditions de productions de chaque énoncé cité dans cette étude. Pour faciliter le repérage des exemples, nous les avons surlignés en jaune.

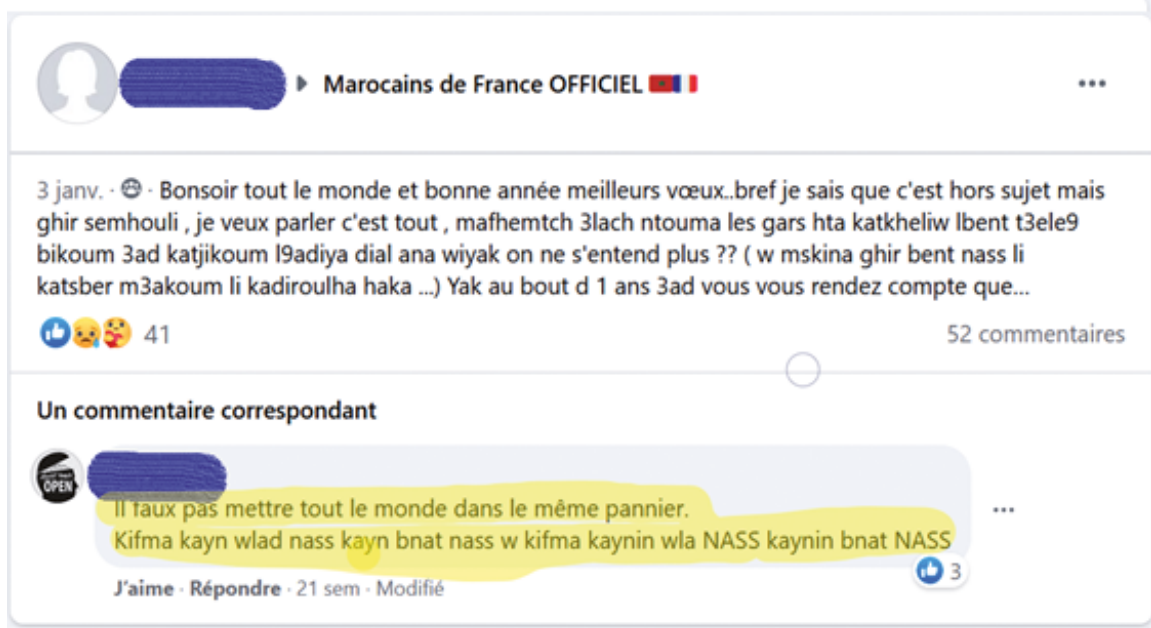

\author{
Illustration 1
}

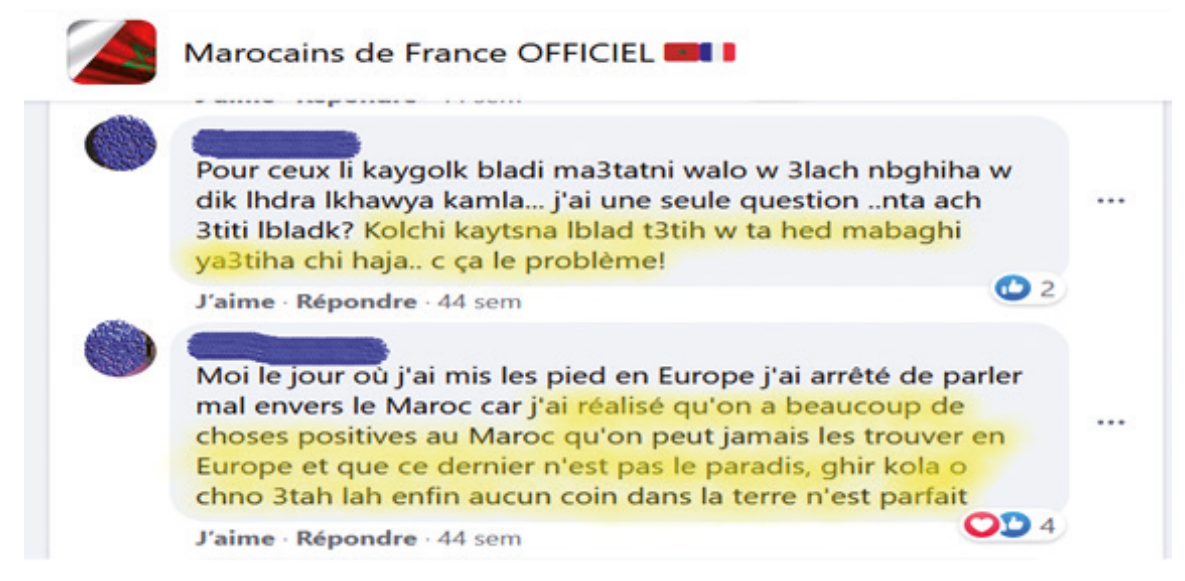

Illustration 2 
Anales de Filología Francesa, n. ${ }^{\circ}$ 29, 2021

L'ARABE MAROCAIN AU CONTACT DU FRANÇAIS SUR LES RÉSEAUX SOCIAUX NUMÉRIQUES...

3 janv. $\cdot \bullet \cdot$...t3eleg bikoum 3ad katjikoum I9adiya dial ana wiyak on ne s'entend plus ?? ( w mskina ghir bent nass li katsber m3akoum li kadiroulha haka ...) Yak au bout $d 1$ ans 3 ad vous vous rendez compte que makatfahmouch avec une fille, lah ykhelikoum vous avez vos soeurs wa fekrouu fihoum y9der yw9e3 lihoum la même choses, stop ...arrêtez de jouer avec les sentiments des autres, je parle...

Illustration 3

Marocains de France OFFICIEL
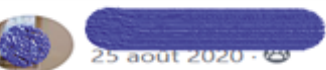

Salam alaykum Mes frères er soeurs Imaghrba. Je viens solliciter votre aide aujourd'hui car je ne sais plus quoi faire. J'ai retarder mes vacances cette année par rapport au covid et j'ai pris le mois de septembre. Bghit nzel Imghrib la semaine prochaine ana w ma mere pour 1 mois et je voulai savoir s'il y' a un risque de fermetures des frontiéres entre le Maroc et la France men hna la fin du mois de septembre. Hna aslan kan sekno $f$ tanja mais tanger tout est fermer alors bghina nezlo $f$ hotel a agadir au moins le nombre de cas machi par rapport à casa tanger marrakech etc. Si quelqu'un a une infos je suis completement perdu. Bonne journée à vous.

\section{Illustration 4}

Marocains de France OFFICIEL
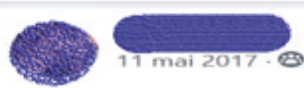

Bghit nswlkom waxh ila drt réservation d vol w apres j'ai un empêchement wach $j$ ai I droit bach $n$ annule I ? Et est ce que I annulation est gratuite I ?

(c) 1

4 commentaires

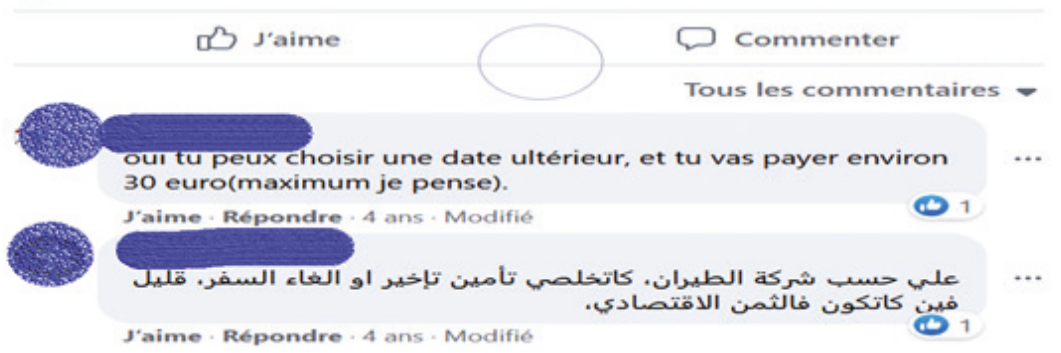

Illustration 5 
Anales de Filología Francesa, n. ${ }^{\circ}$ 29, 2021

MOURAD EL BAROUDI

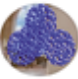

D Marocains de France OFFICIEL

25 août 2020 - $\cdot$ - Salam alaykum Mes frères er soeurs Imaghrba. Je viens solliciter votre aide aujourd'hui car je ne sais plus quoi faire. J'ai retarder mes vacances cette année par rapport au covid et j'ai pris le mois de septembre. Bghit nzel Imghrib la semaine prochaine ana w ma mere pour 1 mois et je voulai savoir s'il y' a un risque de fermetures des frontiéres entre le Maroc et la France men hna la f...

8

99 commentaires

\section{Un commentaire correspondant}

33 Moi personnellene

Moi personnellement je n'irai pas cette année c pas le moment in chaalah l'année prochaine si dieu le veut je nait pas envie d'être enfermé au Maroc

J'aime - Répondre - 39 sem

\section{Illustration 6}

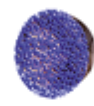

Marocains de France OFFICIEL

16 mars 2018 - ․․ - Salam aleikom , (2) J'ai remarqué une chose que certains marocains nakrine aselhom ,ta9afethom o mnine jayine I darajat ana chi wahdine kay7awlou ydirou mejhoud kbir bech ybedlou lahja dyalhom bech ybanou classe machi 3ib Iwahed ybedel I osloub dyalou aw tari9a dyalou f I7ayat walakine machi I darajat anaka tkoune jay men sebt ghzoula wela men soug larbe 3 o thdar b lahja I...

(b) 115

91 commentaires

\section{Un commentaire correspondant}

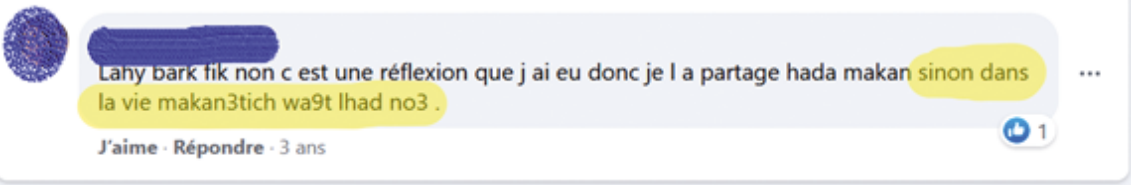

\section{Illustration 7}

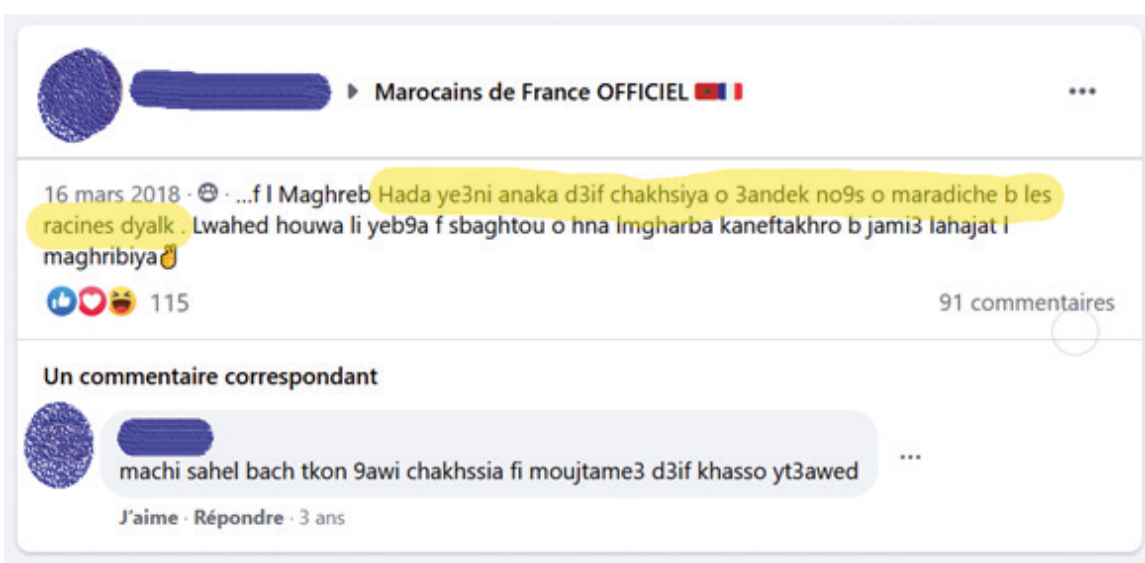

\section{Illustration 8}


Anales de Filología Francesa, n. ${ }^{\circ}$ 29, 2021

L'ARABE MAROCAIN AU CONTACT DU FRANÇAIS SUR LES RÉSEAUX SOCIAUX NUMÉRIQUES...

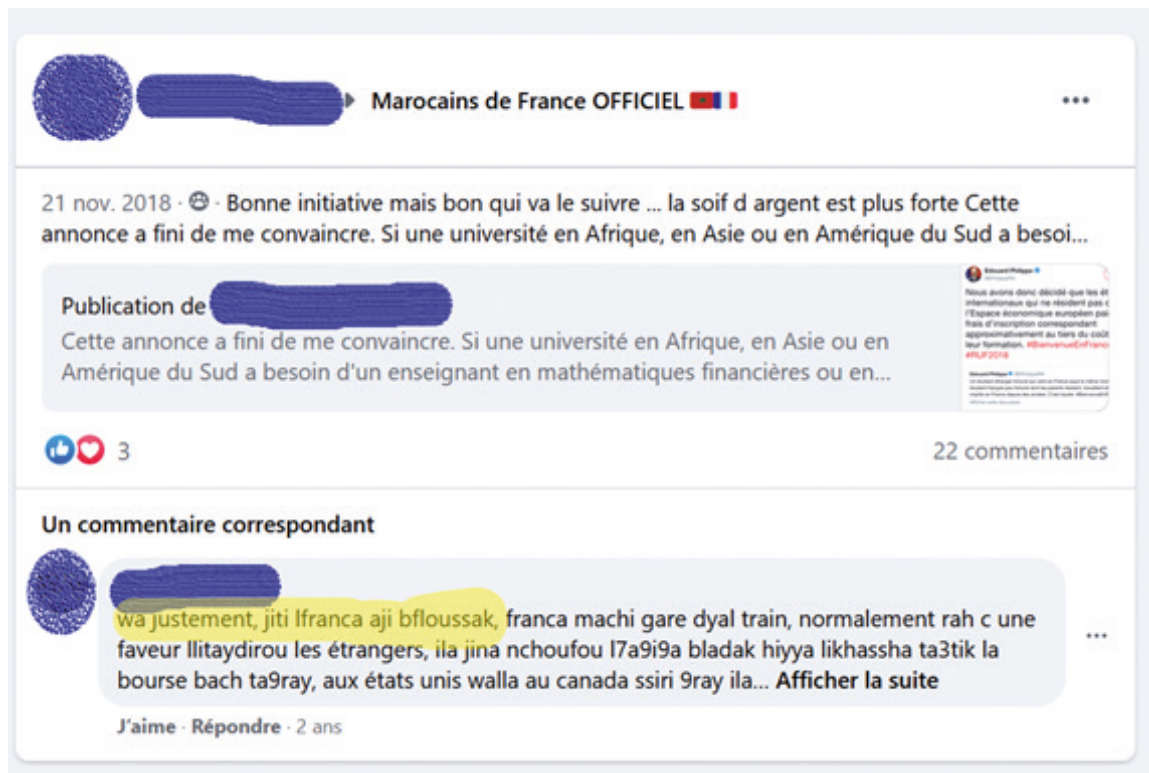

\section{Illustration 9}

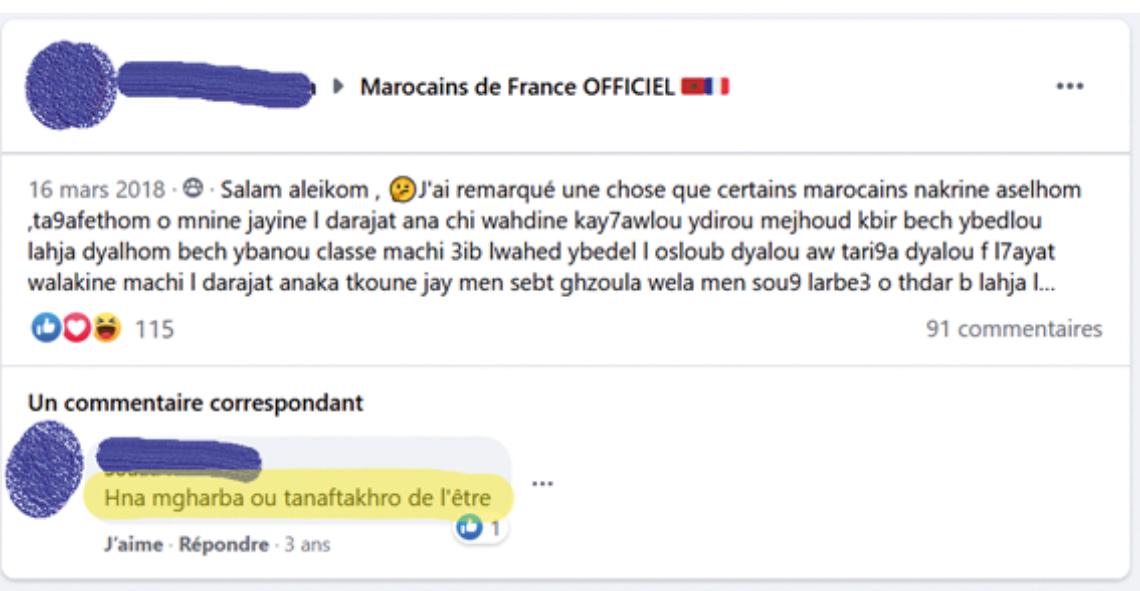

Illustration 10 


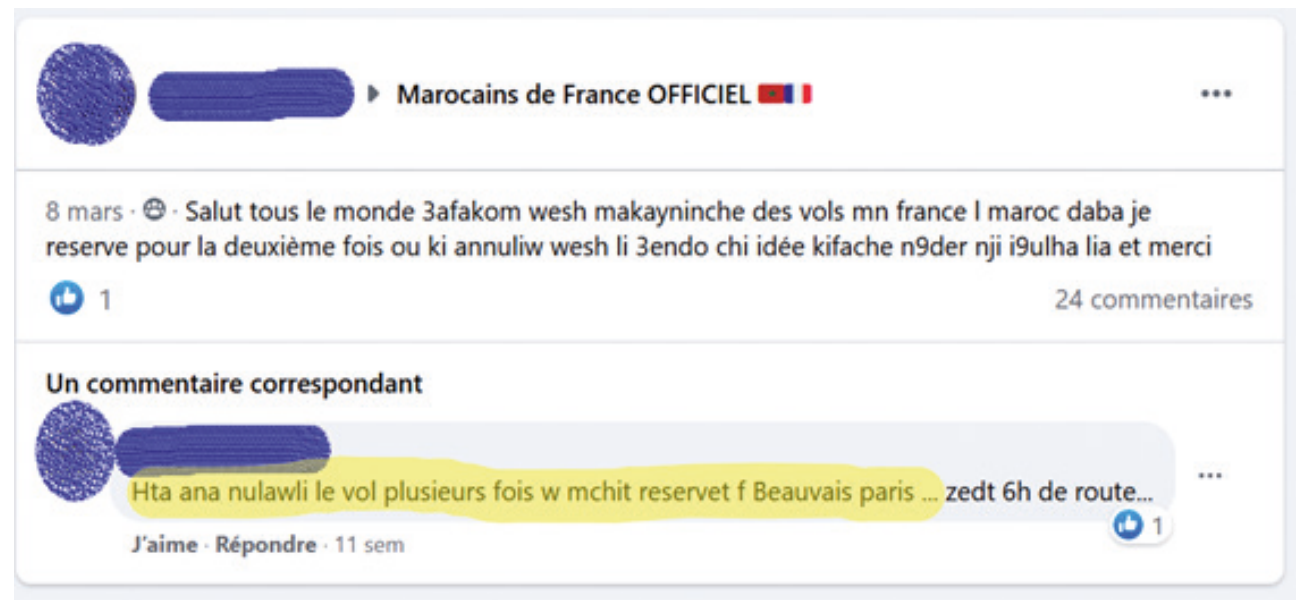

Illustration 11

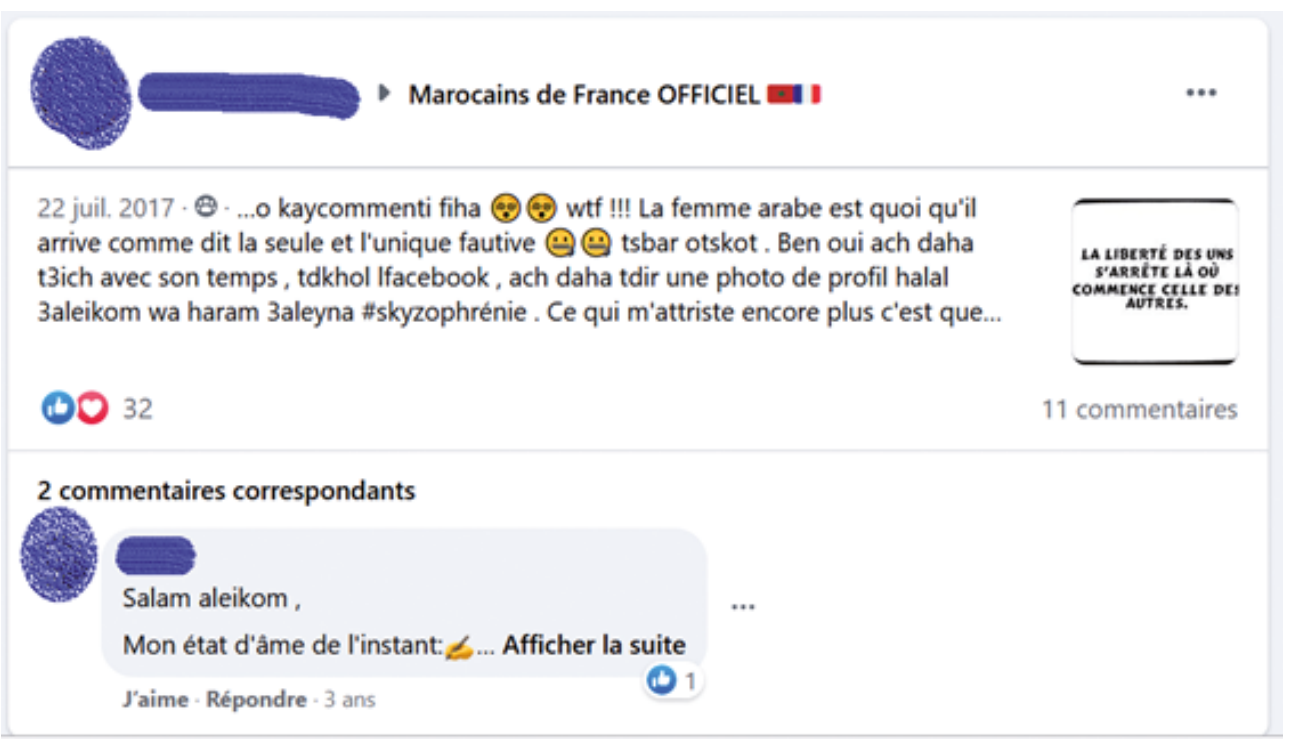

Illustration 12 
